\title{
EXACT MONTE CARLO CALCULATIONS FOR FERMIONS ON A PARALLEL MACHINE
}

\author{
Shiwei Zhang and M.H. Kalos \\ Laboratory of Atomic and Solid State Physics \\ and \\ Center for Theory and Simulation in Science and Engineering \\ Cornell University \\ Ithaca, New York 14853, USA
}

\begin{abstract}
We describe how a recently published algorithm — which addresses the sign problem within the context of the Green's function Monte Carlo method - can be implemented in a parallel distributed environment. The method of parallelization maintains large granularity and therefore low overhead. Despite the stochastic nature of the algorithm, good load-balancing can be accomplished and reproducibility is ensured.
\end{abstract}




\section{INTRODUCTION}

The Green's function Monte Carlo ${ }^{[1],[2],[3],[4]}$ (GFMC) method has been successfully applied to many systems in different areas of physics and has proved an extremely powerful tool in studying quantum many-body problems. The approach allows one to treat with Monte Carlo (MC) explicitly many-dimensional integral equations that result from the Schroedinger equation. Based on an iterative random walk process, it can project out the state with the lowest energy, in principle, for any given symmetry. Here by projecting out, it is meant that the random walk devised from the integral representation of the Schroedinger equation yields asymptotically a collection of configurations distributed according to the wavefunction of the lowest energy state with the given symmetry, starting from essentially arbitrary configurations. Numerous highly accurate and effective calculations have been performed along these lines for many very different systems, mostly of bosons.

Due to the requirement of antisymmetry on the wavefunctions and the nature of Monte Carlo methods, however, a sign problem arises when the method is applied to fermion systems. The positive and negative parts of the wavefunction are represented by independent populations of discrete configurations. Asymptotically the bose ground state dominates and the two independent populations both converge to this symmetric state. So the MC signal of the fermion state, which is the difference of the two populations, is lost in noise. In fact this problem is encountered almost universally in Quantum Monte Carlo treatment of fermion systems, possibly in slightly different forms. It has remained unsolved despite the existence of various methods that yield accurate and useful results.

We have recently proposed an algorithm ${ }^{[5],[6],[7]}$ with the Green's function Monte Carlo method to treat the sign problem exactly for small systems of fermions. In this paper, we outline the essential ingredients of the algorithm and then describe our implementation of the algorithm in a parallel distributed environment on a coupled cluster of workstations. The parallel implementation is novel and aimed at the fermion algorithm. It is technically rather simple and flexible and serves very well the purpose of testing and developing the algorithm. On the other hand, it attempts to address some issues that are likely to come up in 
porting any typical GFMC codes to parallel machines and thus the parallelization scheme is by no means limited to the particular algorithm.

\section{DESCRIPTION OF THE FERMION ALGORITHM}

We first briefly describe the fermion algorithm with the GFMC method in the context of small atoms. The nucleus is assumed to be fixed at the origin. In atomic units, the many-body Schroedinger equation can be written as

$$
\left[-\frac{1}{2} \nabla_{\mathbf{X}}^{2}+V(\mathbf{X})\right] \phi(\mathbf{X})=E \phi(\mathbf{X})
$$

where $\mathbf{X}$ is a $3 N$ dimensional vector denoting the coordnates of all $N$ electrons in $3 \mathrm{D}$ real space. The term $V$ denotes the Coulomb potential of the system. The wavefunction is $\phi$ and the energy $E$ is negative. We can change the length scale of the system by $\mathbf{R}=\sqrt{2|E|} \mathbf{X}$. Noticing that the Coulomb potential $V$ scales as $1 /$ length, we can then transform Eq (1) to

$$
\left(-\nabla_{\mathbf{R}}^{2}+1\right) \psi(\mathbf{R})=-\sqrt{\frac{2}{|E|}} V(\mathbf{R}) \psi(\mathbf{R}) .
$$

We note that the rescaling allows us to work with $\mathbf{R}$ in solving for the ground state wavefunction without knowing $E$ and conveniently evaluate the energy at the end with samples whose distribution is the rescaled wavefunction $\psi$. Eq (2) can be rewritten as an integral equation as follows:

$$
\psi(\mathbf{R})=\lambda \int d \mathbf{R}^{\prime} G\left(\mathbf{R}, \mathbf{R}^{\prime}\right)\left[-V\left(\mathbf{R}^{\prime}\right)\right] \psi\left(\mathbf{R}^{\prime}\right),
$$

where $G\left(\mathbf{R}, \mathbf{R}^{\prime}\right)$ is the Green's function for the operator $\left(-\nabla_{\mathbf{R}}^{2}+1\right)$ and has a known analytical form ${ }^{[2],[7],[8]}$. It is everywhere positive and is normalized to 1 . The eigenvalue $\lambda$ is $\sqrt{\frac{2}{|E|}}$.

In principle, $\mathrm{Eq}(3)$ can be solved by iteration for the eigenfunction corresponding to the lowest eigenvalue $\lambda$ for a given symmetry. In other words, we can arrive at the lowest energy state of a given symmetry by applying repetitively the iterative relation from an arbitrary initial function having non-zero overlap with this state. Thus the problem of solving the partial differential equation 
(2) is reduced to doing many-dimensional integrals, something natural to MC methods. This forms the basis of the GFMC approach.

In practice, the iteration process is carried out with the wavefunction in each generation represented by a collection of individual configurations, or random walkers. The walkers live in configuration space and they move from one point $\mathbf{R}^{\prime}$ in this space to another $\mathbf{R}$ according to the probability distribution function $G\left(\mathbf{R}, \mathbf{R}^{\prime}\right)$. Thus the iteration becomes a random walk in configuration space with a population of individual configurations as walkers in each step. The function $-V$ in $\mathrm{Eq}(3)$ is treated as a multiplicative weight or as a source of branching of walkers. Asymptotically the random walk yields a distribution of walkers that samples the wavefunction $\psi$ with the lowest energy of a given symmetry. With sufficiently many walkers drawn from the asymptotic distribution, information can be extracted on the properties of the state, such as the energy.

Importance sampling ${ }^{[1],[4]}$ is a powerful method in reducing the fluctuations in the random walk. The integral equation (3) can be transformed to obtain

$$
i(\mathbf{R}) \psi(\mathbf{R}) \propto \int d \mathbf{R}^{\prime}\left[i(\mathbf{R}) G\left(\mathbf{R}, \mathbf{R}^{\prime}\right) / i\left(\mathbf{R}^{\prime}\right)\right]\left[-V\left(\mathbf{R}^{\prime}\right)\right] i\left(\mathbf{R}^{\prime}\right) \psi\left(\mathbf{R}^{\prime}\right)
$$

The random walk is now carried out according to $\mathrm{Eq}$ (4) to sample a different distribution $i \psi$. The importance function $i(\mathbf{R})$ is the asymptotic contribution of a walker starting at R. For a system of bosons, that is just the desired ground state wavefunction $^{[1],[4]}$ and therefore a trial wavefunction can be used for $i$.

Naive implementation of the GFMC method as described above to fermion systems, however, quickly leads to the sign problem. For a system of fermions, the wavefunction must change sign under interchange of particles with like spins. To represent such an antisymmetric wavefunction, an equal number of positive and negative walkers are needed. In the above algorithm, the two populations are not distinguished and are therefore asymptotically identical. This leads to a zero $\mathrm{MC}$ estimate of $\psi$ coupled with random fluctuations and thus the decay of signal to noise ratio. In other words, there exists a "super-symmetry" between the positive and negative populations in the above algorithm and both will be symmetric in the long-term. So the method is not stable.

To overcome these difficulties, our new fermion algorithm has the following 
ideas: (i) keep equal numbers of positive and negative walkers and introduce a "repulsion" between them to separate the two populations; and (ii) introduce different importance functions for positive and negative walkers to break the "super-symmetry". Below we shall describe the actual formalism ${ }^{[6],[7]}$ based on these ingredients.

The sign problem can in fact arise from yet another source in the current form of the GFMC approach, namely negative values of the potential term $-V$. But since it is dealt with rigorously in the fermion approach, this additional source does not pose any special difficulty. The antisymmetric wavefunction can be written as

$$
\psi(\mathbf{R})=\psi^{+}(\mathbf{R})-\psi^{-}(\mathbf{R})
$$

where $\psi^{ \pm}(\mathbf{R})$ map into each other by any odd permutation of like spins. We shall use $\psi^{+}$to denote the positive part of the antisymmetric wavefunction and $\psi^{-}$the absolute value of the negative part and rewrite $(3)$ in a coupled way:

$$
\psi^{ \pm}(\mathbf{R})=\lambda \max \left\{0, \pm \int d \mathbf{R}^{\prime} G\left(\mathbf{R}, \mathbf{R}^{\prime}\right)\left[-V\left(\mathbf{R}^{\prime}\right)\right]\left(\psi^{+}\left(\mathbf{R}^{\prime}\right)-\psi^{-}\left(\mathbf{R}^{\prime}\right)\right)\right\} .
$$

We now introduce a transformed wavefunction

$$
\tilde{\psi}^{ \pm}(\mathbf{R})=V^{\prime}(\mathbf{R}) i^{ \pm}(\mathbf{R}) \psi^{ \pm}(\mathbf{R})
$$

where $V^{\prime}(\mathbf{R})$ is positive and symmetric and $i^{ \pm}(\mathbf{R})$ map into each other like $\psi^{ \pm}(\mathbf{R})$. Letting $\tilde{G}\left(\mathbf{R}, \mathbf{R}^{\prime}\right)=V^{\prime}(\mathbf{R}) G\left(\mathbf{R}, \mathbf{R}^{\prime}\right)$ and $W(\mathbf{R})=-V(\mathbf{R}) / V^{\prime}(\mathbf{R})$, we can write (6) in terms of the transformed wavefunction:

$$
\tilde{\psi}^{ \pm}(\mathbf{R})=\lambda i^{ \pm}(\mathbf{R}) \max \left\{0, \pm \int d \mathbf{R}^{\prime} \tilde{G}\left(\mathbf{R}, \mathbf{R}^{\prime}\right) W\left(\mathbf{R}^{\prime}\right)\left[\frac{\tilde{\psi}^{+}\left(\mathbf{R}^{\prime}\right)}{i^{+}\left(\mathbf{R}^{\prime}\right)}-\frac{\tilde{\psi}^{-}\left(\mathbf{R}^{\prime}\right)}{i^{-}\left(\mathbf{R}^{\prime}\right)}\right]\right\} .
$$

We will now attempt to solve for $\tilde{\psi}^{ \pm}$by MC by representing the antisymmetric solution at any stage as

$$
\tilde{\psi}(\mathbf{R})=\sum_{k} s_{k} \delta\left(\mathbf{R}-\mathbf{R}_{k}\right)
$$

with $k$ being the label of walkers and $s_{k}$ the sign of walker $k$, i.e., $s_{k}= \pm 1$ for positive/negative walkers. 
The function $W(\mathbf{R})$ is associated with branching in the random walk. So it is an undesirable feature that the Coulomb potential is singular in certain regions in the configuration space. We can regulate the behavior of weights by choosing proper forms for $V^{\prime}$. One simple construction is to take $V^{\prime}$ as the summation of the absolute values of all the terms in $V$. Then the weight $W(\mathbf{R})$ in $(8)$ is well-behaved and the kernel of the form $\tilde{G}\left(\mathbf{R}, \mathbf{R}^{\prime}\right)=\frac{1}{\left|\mathbf{r}_{i}\right|} G\left(\mathbf{R}, \mathbf{R}^{\prime}\right)$ can be sampled directly ${ }^{[8]}$. (The other kernel $\frac{1}{\left|\mathbf{r}_{i}-\mathbf{r}_{j}\right|} G$ can be transformed to the same form via a spatial rotation in $3 N$ dimensions.)

The function $i$ plays the role of a one-body force which acts differently on positive and negative walkers to break the super-symmetry that exists between them, which can be seen from the fact that positive and negative walkers can be exchanged while the distribution remains a solution to the integral equation. It reflects the asymptotic contribution to the solution of a walker at a certain position ${ }^{[7]}$. Qualitatively, the importance function $i^{+}(\mathbf{R}) \quad(>0)$ for positive walkers should have the effect of biasing the walker toward regions in configuration space where the wavefunction is positive. Thus where the wavefunction is likely to be negative, it falls off very rapidly due to the high population of negative walkers. The importance function for negative walkers is also positive and is related to $i^{+}(\mathbf{R})$ by $i^{-}(\mathbf{R})=i^{+}(P \mathbf{R})$, where $P$ denotes an odd number of interchanges of like spins. The importance function is used primarily to filter out "preferred positions" for walkers. It is desirable that we extract as much as possible information about the nodes, from an antisymmetric trial wavefunction and build it into the importance function.

In our sampling process, the whole ensemble of walkers will be considered to generate every new walker for the next step. This interaction is crucial in preserving the fermion component of the wavefunction at each stage. According to Eqs (8) and (9), we will sample positions for new walkers from the density distribution

$$
K\left(\mathbf{R},\left\{\mathbf{R}^{\prime}\right\}\right)=\sum_{k} s_{k}^{\prime} \tilde{G}\left(\mathbf{R}, \mathbf{R}_{k}^{\prime}\right) W\left(\mathbf{R}_{k}^{\prime}\right) / i\left(\mathbf{R}_{k}^{\prime}, s_{k}^{\prime}\right)
$$

where $\left\{\mathbf{R}^{\prime}\right\}$ denote the current ensemble of walkers and $i(\mathbf{R}, \pm 1)=i^{ \pm}(\mathbf{R})$. The density $K$ is as often negative as positive for the ground states of fermion systems. 
The important point here is that we shall sample $K$ directly rather than sampling walkers from each term in $K$. This can be accomplished by a rejection method ${ }^{[9]}$ : First sample from $K^{\prime}\left(\mathbf{R},\left\{\mathbf{R}^{\prime}\right\}\right)=\sum_{k}\left|\tilde{G}\left(\mathbf{R}, \mathbf{R}_{k}^{\prime}\right) W\left(\mathbf{R}_{k}^{\prime}\right) / i\left(\mathbf{R}_{k}^{\prime}, s_{k}^{\prime}\right)\right|$ and then use $|K| / K^{\prime}$ to accept the new walker. The newly sampled walker $\mathbf{R}$ is assigned a minus sign if $K$ is negative.

The importance function in front in $\mathrm{Eq}$ (8) can be dealt with by, say, another rejection. For example, we can scale the importance function such that its maximum is 1 and then use $i$ directly as acceptance probabilities. Equal and constant numbers of positive and negative walkers are maintained throughout the calculation. The MC bias introduced by this is corrected by running with different numbers of walkers $M$ and fitting the energy vs. $1 / M$ curve to polynomials and extrapolating to $M=\infty$.

With an ensemble of walkers $\left\{\mathbf{R}_{k}\right\}$ that samples $\tilde{\psi}$ and a test wavefunction $\psi_{T}(\mathbf{R})$, the energy eigenvalue $E$ for the system can be easily calculated:

$$
\sqrt{\frac{2}{|E|}}=\frac{\sum_{k=1}^{M}\left(-\nabla^{2}+1\right) \psi_{T} /\left.V^{\prime} i^{+}\right|_{\mathbf{R}_{k}^{+}}-\sum_{k=1}^{M}\left(-\nabla^{2}+1\right) \psi_{T} /\left.V^{\prime} i^{-}\right|_{\mathbf{R}_{k}^{-}}}{\sum_{k=1}^{M} \psi_{T} W /\left.i^{+}\right|_{\mathbf{R}_{k}^{+}}-\sum_{k=1}^{M} \psi_{T} W /\left.i^{-}\right|_{\mathbf{R}_{k}^{-}}} .
$$

The importance functions used in our calculations are derived from the trial wavefunction $\psi_{T}$. They are constructed in a rather crude fashion based on the following guidelines: In the region with $\psi_{T}>0$, far away from the nodal surface (as given by $\psi_{T}$ ), the importance function for a positive walker should approximate the trial function; It should decrease to some small but non-zero value as the nodal surface is approached from the positive region; And it should continue to decrease and become vanishingly small deep into the negative region of the trial wavefunction. For example, to study the 1s2s excited state of the He atom and the ground state of the $\mathrm{Li}$ atom, the importance functions are both based on the Hartree-Fock wavefunctions which give $r_{1}=r_{2}$ as the nodal surface, where 1 and 2 label the two electrons with parallel spins and $r_{1}$ and $r_{2}$ are their respective distances from the nucleus. They have the form

$$
i^{+}(\mathbf{R}) \propto \frac{1}{1+a e^{-\alpha\left(r_{2}-r_{1}\right)}} I_{\text {orbit }}(\mathbf{R})
$$

where $a$ and $\alpha$ are parameters. The last term $I_{\text {orbit }}$ is a function to anchor the electrons to corresponding orbitals and prevent them from drifting away to 
infinity from the nucleus. The importance function $i^{-}$is obtained from the above by an odd permutation as described before. When $\alpha$ is positive, $i^{+}$clearly favors configurations with $r_{1}<r_{2}$ while $i^{-}$favors $r_{1}>r_{2}$, thus satisfying our prescription for the importance functions. The trial wavefunctions we used for small atoms are those of Umrigar, Wilson and Wilkins ${ }^{[10]}$.

The above algorithm has been applied to test systems including small atoms (up to the ground state of the Nitrogen atom). The method is stable and yields answers to the energies that agree well with experiment ${ }^{[6]}$. As the system size grows, the dimensionality increases in which the random walkers reside. So among other difficulties, the walker "density" decreases for fixed number of walkers. The effect of repulsion between positive and negative walkers and the cancellation will diminish. It is possible to construct convolutions of the Green's functions to enhance the range of interaction. But it is not so clear what the scaling behavior will be with all these elements. However, regardless of the scaling, it is clear the availability of scalably parallel computers will help in extending the method to larger systems, and indeed to assess the scaling of the algorithm itself, as the number of walkers can be greatly increased to increase stability. We are now making that study. As we shall demonstrate below, the granularity of these calculations is normally high and therefore the parallelism scales well.

\section{PARALLEL IMPLEMENTATION OF THE ALGORITHM}

In this section, we describe our implementation of the above method on parallel distributed computers. We first summarize (and reorganize slightly) the algorithm in such a way that it becomes more transparent how the parallelization should be carried out:

1). Generate $M$ walkers by Metropolis from a test wavefunction or randomly.

2). Evaluate $p\left(\mathbf{R}^{\prime}\right) \propto \mathcal{N}\left(\mathbf{R}^{\prime}\right) W\left(\mathbf{R}^{\prime}\right) / i^{+}\left(\mathbf{R}^{\prime}\right)$ for every walker $\mathbf{R}_{i}^{+}$, where $\mathcal{N}\left(\mathbf{R}^{\prime}\right)$ $=\int V^{\prime}(\mathbf{R}) G\left(\mathbf{R}, \mathbf{R}^{\prime}\right) d \mathbf{R}$. Then normalize them such that $\sum_{i} p_{i}=1$.

3). Pick walker $i$ with probability $p_{i}$. Sample a new configuration $\mathbf{R}$ from either $\mathbf{R}_{i}^{+}$or $\mathbf{R}_{i}^{-}$(with probability $1 / 2$ ) according to the kernel $V^{\prime}(\mathbf{R}) G\left(\mathbf{R}, \mathbf{R}_{i}^{ \pm}\right)$.

4). For the proposed new walker $\mathbf{R}$, compute the ratio $|K| / K^{\prime}$ and continue on with this probability (else go back to 3 ). 
$5)$. Compute the importance function value for $\mathbf{R}\left(i^{+}(\mathbf{R})\right.$ if $K>0$, and $i^{-}(\mathbf{R})$ if $K<0$.) and use this as acceptance probability to accept $\mathbf{R}$ as a new walker. Determine the sign of the new walker from the sign of $K$.

6). Repeat 3) - 5) till $M$ positive walkers and $M$ negative walkers have been obtained. Positive and negative walkers are related by odd permutations, so whenever a walker is accepted both populations are increased. An odd permutation indicates interchanging an odd number of times pairs of electrons with parallel spins. It can in principle be done randomly among all possibilities. A more systematic way is to inherit the permutation relation of the parent walker and its opposite-sign walker in the previous generation.

7). Steps 2) through 6)complete one generation (one step of the random walk) and the process is iterated, or the random walk advanced.

We can start collecting data (e.g., evaluating the eigenvalue) after the iteration has converged. To reduce the variance of the $\mathrm{MC}$ estimators from this process, we need as many uncorrelated walkers as possible in the asymptotic regime of the iteration.

There exist various options to accomplish the above and therefore reduce the statistical fluctuation. The most straightforward among these is to repeat the whole procedure (steps 1 through 7 ) many times with different random number streams. In terms of parallel computing, this implies that we run independent replicas of the main part of the program on different processors. This approach requires very little effort in parallelizing the code and has essentially no overhead cost. Only a minimum amount of communication is required between processors at the beginning of the computation to assign different random number generator (RNG) seeds to different replicas, and also at the end to combine the data and carry out the final analysis. This intrinsic nature of many $\mathrm{MC}$ calculations makes parallelization extremely effective, although trivial. And in some cases, this is indeed the most efficient approach. In our calculations, for small numbers of walkers it is rather cheap to carry out many generations, so the relaxation time (the time between the initial generation and where the walker distribution has equilibrated and represents the one being sampled) is relatively short. Then the replica scheme can be employed to yield close to ideal speed-up. 
The procedure of running different replicas, however, may not be practical as the number of walkers increases, or when the convergence time is long. Since the number of walkers is fixed in every run in our calculations, it is necessary to do runs with different numbers of walkers $M$ and extrapolate to the infinite $M$ limit to account for the population control bias. The amount of computation in the bulk part of the calculation (steps 3-5) scales almost as $M^{2}$, so the convergence time (measured by, say, cpu time) will increase significantly if we choose large values for $M$. In this case, it becomes either inefficient or impossible to apply the replica approach. It is then necessary to spread a single run onto different processors, i.e., parallelize the code at the next level.

We shall next mention briefly the issue of reproducibility before discussing our implementation of this level of parallelism. Despite the random nature of MC simulations, reproducibility has always been a desirable feature in a practical sense. It is especially important in testing a $\mathrm{MC}$ code after any modifications (either in the code itself or in the running environment). In other words, at any experimental stage of a MC calculation, it is highly desirable that a calculation yield the same results when repeated with the same RNG seeds. For example, in porting the code to parallel machines, it is extremely helpful if the calculation can be independent of the number of processors and reproducible even from serial to parallel environments. This can be trivially satisfied in the independent replica approach discussed above, by assigning a different RNG stream to each different replica. In our parallel implementation below, attention is paid to ensure reproducibility.

Our calculations are carried out on a coupled cluster of IBM RS6000's at the Cornell Theory Center, using PVM, a message-passing environment for parallel distributed computing. The basic scheme developed, however, will easily apply to shared memory environments as well.

As is true for many $\mathrm{MC}$ calculations, the memory requirement here is fairly small and all the memories involved can be readily stored in each and every processor. The generation of new walkers (including the rejection processes) is by far the major part of the computation. One key observation is that within each iteration, new walkers can be generated independently of each other except 
for the RNG stream. In other words, the repetition of steps 3 through 5 can be partitioned and executed in parallel provided that the seemingly sequential dependence of the RNG stream is addressed properly. We emphasize that due to the interaction among all walkers in this algorithm, the usual way to parallelize a random walk process, namely to partition the current population onto different processors each of which advances its own fraction of random walkers, leads to larger bias and is therefore undesirable. The particular form of walker interaction that arises in this algorithm is novel, and requires a novel approach to the partitioning, which we have described. Similarly the more "natural" approach to follow the sequential code and parallelize the computation of the interactions (which consumes the most time in the calculation) for generating each new walker would have much overhead from message-passing.

We use one processor as the host node and $n_{\mathrm{pr}}$ other processors as computing nodes. In each iteration of the random walk, we send to and store in each processor all relevant information about all walkers in the old population as well as the relative weights $p_{i}$. Then $n_{\mathrm{thr}}\left(\geq n_{\mathrm{pr}}\right)$ parallel threads are defined. Each of these has a particular RNG seed attached to it and repeats steps 3 to 5 until $M / n_{\text {thr }}$ new walkers have been produced. With $n_{\text {thr }}$ independent RNG streams and an arbitrarily chosen way to correspond threads to labels of the respective groups of new walkers they produce, these threads can be executed in parallel in any order, with the result completely deterministic. This deterministic nature guarantees that the procedure is reproducible as a parallel process or by a serial code using the same thread to walker label correspondence set (arbitrarily) above. After a processor has finished the assigned threads in one iteration, all necessary preparations, such as the computations of the unnormalized weights $p$, are carried out for the new walkers generated. The information about these new walkers is then sent back to the host, which normalizes the relative weights $p_{i}$ upon completion of processes on all computing nodes. The new population of walkers can now be viewed as the "old population" and the procedure described above is repeated for the next iteration. As we can see, the granularity in these calculations is normally very large under the current scheme. Obviously, the normalization of $p$ by the host can in principle be made parallel as well, 
but the amount of computation is negligible relative to the bulk part and the parallelization is not pursued.

To reduce the overhead cost in communication, it is desirable to have $n_{\mathrm{thr}}=$ $n_{\mathrm{pr}}$, such that message passing only takes place between iterations. The number of times steps 3 to 5 are repeated in each thread may vary and is unknown $a$ priori, due to the rejection procedures involved in the sampling. But the current scheme partitions a job stochastically into $n_{\mathrm{pr}}$ equal pieces. Since the number of new walkers each thread has to produce is large and all the threads generate new walkers from an identical source, the threads are intrinsically well balanced in computing loads. In fact, if we assume the cpu time to generate each walker is $t_{1}$, and the fluctuation in this time $\sigma_{1}$, then in each step of the random walk, the loads of processors should have a relative fluctuation of about $\frac{\sigma_{1}}{t_{1}} \frac{1}{\sqrt{M_{\mathrm{pr}}}}$, where $M_{\mathrm{pr}}=M / n_{\mathrm{pr}}$ is the number of walkers each processor needs to produce. The number $M_{\mathrm{pr}}$ is generally more than 100 to maintain large granularity. So when all the processors are equally available, it is reasonable to expect this to give high performance. To guarantee load balancing or deal with uneven level of availability of different processors, we can make $n_{\text {thr }}$ several times as large as $n_{\mathrm{pr}}$ (or larger), and feed processors with threads one by one. Of course, there will be some trade-off between granularity and load-balancing. The larger $n_{\text {thr }}$ is, the more load-balancing can be achieved. On the other hand, the overhead cost increases as more threads are used and more message-passing conducted. Since load balancing is in general quite easily obtained in this application, to maintain larger grain size is more important to the efficiency of the calculations.

In the above discussion, we have implicitly assumed the availability of many long RNG sequences, adequately independent of each other. The issue of independent pseudo RNG streams on MIMD machines has been addressed by Percus and $\mathrm{Kalos}^{[11]}$. A simple scheme was proposed to generate large numbers of independent pseudo-random number sequences. The number of such sequences that the RNG is capable of generating is far more than adequate for our purposes. In fact only $n_{\mathrm{thr}}$ (or $n_{\mathrm{thr}}+1$, with an extra and different stream for the host code in generating initial configurations, etc) streams are needed in our calculations. Another important fact we have employed about the RNG is that it is easily 
portable from processor to processor by keeping track of the values of the seeds.

In most of our calculations, the host evaluates the eigenvalue estimators from the old population and carries out other data analysis while the computing nodes are working on the next iteration. In this arrangement the host has a very moderate load, well-suited to the set-up of the cluster we used, which has a couple machines serving as interactive nodes while others as computing nodes. One could, of course, assign threads to the host as well and attach the computation of eigenvalue estimators to the threads. This way each processor evaluates the estimators from the new walkers it has generated and includes them in the information sent to the host. The host will then only have to carry out the very small amount of computation in data analysis, either between iterations or at the end of the whole calculation.

We have run the Be code with $M$ around 1000 on four to ten processors for more than 10000 steps. Each step (iteration) in such a calculation would take more than a minute on a single processor. So the frequency of communication is relatively low. Of course, in the process of experimenting with the algorithm, different combinations of these numbers are used and the grain-size can vary significantly. But the relative flexibility of our scheme enables rather efficient parallelization in most cases. For example, one can combine the above with the replica approach as the number of processors becomes large to maintain efficiency. In other words, the above process can be run on a fraction of all the processors and replicas of such processes can be created to occupy the whole system so that the calculation remains coarse-grained. With the better wavefunctions in Ref [10], we have obtained a ground state energy $-14.66692 \pm 0.00057$ Hartrees for the Be atom, compared to the experimental value of -14.6673 Hartrees, and $-54.567 \pm 0.033$ Hartrees for the ground state of $\mathrm{N}$, compared to the experimental value of -54.590 Hartrees.

As pointed out before, the memory requirement in this algorithm is rather small. So with a speed-up around $80 \%$ (i.e., the factor by which wall-clock time is reduced is about $80 \%$ the number of compute nodes) accomplished in the current scheme, the cluster environment is well suited for these calculations. The improvement of a factor of 8 (64 in cpu time) in the accuracy of the Be result 
reported above over that in Ref [6] is a result of both a better trial wavefunction and much more computing power from the speed-up. Needless to say, any improvement on the speed of message-passing in PVM would enable even higher efficiency.

\section{ACKNOWLEDGMENTS}

We wish to thank Hugh Caffey and John Zollweg for helpful discussions on PVM. The Cornell Theory Center is funded by the U.S. National Science Foundation, by New York State, by IBM, and by Cornell University. 


\section{REFERENCES}

1. D.M. Ceperley and M.H. Kalos, in Monte Carlo Methods in Statistical Physics, ed. by K. Binder (Springer Verlag, 1979).

2. M.H. Kalos, Phys. Rev. 128, 1791 (1962).

3. K.E. Schmidt and M.H. Kalos, in Applications of the Monte Carlo Method in Statistical Physics, ed. by K. Binder (Springer Verlag, 1984).

4. M.H. Kalos, D. Levesque, and L. Verlet, Phys. Rev. A9, 2178 (1974).

5. M.H. Kalos, in Computational Atomic and Nuclear Physics, ed. by C. Bottcher, M.R. Strayer and J.B. McGrory (World Scientific 1989); M.H. Kalos, J. Stat. Phys. 63, 1269 (1991).

6. S. Zhang and M.H. Kalos, Phys. Rev. Lett. 67, 3074 (1991).

7. M.H. Kalos and S. Zhang, in Recent Progress in Many-body Theories, vol. 3, ed. by C.E. Campbell and E. Krotscheck (Plenum, in press).

8. M.H. Kalos, J. of Comp. Phys. 2, 257, (1967).

9. M. H. Kalos and P. A. Whitlock, Monte Carlo Methods, (Wiley, New York, 1986).

10. C.J. Umrigar, K.G. Wilson and J.W. Wilkins, Phys. Rev. Lett. 60, 1719 (1988); C.J. Umrigar, K.G. Wilson and J.W. Wilkins in Computer Simulation Studies in Condensed Matter Physics: Recent Developments, ed. by D.P. Landau, K.K. Mon and H.B. Schuttler ( Springer Verlag 1988).

11. O. Percus and M.H. Kalos, Journal of Parallel and Distributed Computing $\underline{6}, 477$ (1989). 